\title{
Ajustando o foco atencional de alunos adultos a itens gramaticais opacos: conectivos concessivos
}

\author{
Redirecting adult learners' attentional focus \\ to opaque grammatical items: concessive \\ connectives
}

Ana Lúcia Pitanguy Marques*

Universidade Federal de Minas Gerais - UFMG

Belo Horizonte - Minas Gerais/Brasil

\begin{abstract}
RESUMO: O presente trabalho mostra o cerne - a metodologia - assim como a análise quantitativa e as conclusōes de uma investigação sobre os efeitos promissores do redirecionamento atencional de alunos adultos a aspectos específicos da LE. O trabalho desencadeador da 'percepção consciente' desses alunos à importância e ao uso dos conectivos concessivos em períodos compostos por subordinação ocorreu por meio de uma sequência de intervençōes na prática pedagógica, em atividades /tarefas interligadas tematicamente, que propiciaram uma maior recorrência e saliência do foco de tratamento. Os resultados promissores indicam que essas atividades geradoras de percepção consciente e conscientização foram as maiores responsáveis por uma alteração significativa na escalada positiva do nível de desempenho na produção escrita desses alunos.
\end{abstract}

PALAVRAS-CHAVE: 'percepção consciente', noticing, atenção, foco atencional, foco na forma, conscientização.

ABSTRACT: This paper shows the core - the methodology - as well as the quantitative analyses and promising findings of an investigation on the effects of redirecting adult learners' attentional foci to specific areas of a foreign language - English. The work carried out to trigger learners' awareness and 'noticing' of the concessive connectives in subordinate clauses took place during classroom practice and was implemented through a series of thematically-integrated tasks. Those aimed at exposing learners to a treatment focus that was more recurrent and salient. The findings suggest that activities which triggered the 'noticing' of the treatment foci were the main responsible for the significant changes in the use of the target structures in the written texts, and possibly for the rise of the participants' level of linguistic competence in their written production.

KEY WORDS: noticing, attention, attentional focus, focus on form, awareness.

* anapitanguy@hotmail.com 


\section{Introdução}

O presente trabalho mostra a metodologia empregada na pesquisa de mestrado, ${ }^{1}$ de cunho quantitativo, sobre o papel da percepção consciente de aspectos linguísticos da língua inglesa (LI) por um grupo de alunos adultos noticing. ${ }^{2}$ Destacam-se aqui as etapas da prática pedagógica, os tratamentos e dados obtidos na investigação, que teve como objetivo verificar a possibilidade de alteração no nível de competência linguística dos participantes do grupo de tratamento. Após participarem de uma série de atividades comunicativas, sua produção escrita revelou claros indícios de um aumento significativo da percepção consciente, e ativação, dos conectivos concessivos while, whereas, despite, in spite of, even if, foco do tratamento.

\section{Revisão da literatura}

A investigação teve como pilares teóricos o construto 'percepção consciente' proposto por Schmidt e Frota (1986) e seus desdobramentos em relação ao construto 'atenção' (SCHMIDT, 1990, 2001; ROBINSON, 1995; ELLIS, N., 1999; TOMLIN; VILLA, 1994). As escolhas da prática pedagógica foram orientadas pelas considerações de Doughty (2001), Talmy (2000) e Schmidt (2001) sobre a importância do construto 'atenção' na aquisição de uma segunda língua (L2); enquanto a implementação das atividades seguiu os princípios sugeridos pela abordagem Foco na Forma (FnaF) proposta e definida por Long (1991).

O presente estudo também se apoiou na teoria de Pienemann (1998), que postulou a existência de uma escala ascendente de 'processabilidade linguística' para a aquisição sustentada da língua estrangeira (LE), na qual as orações subordinadas são a última etapa do processo. Referindo-se à língua materna (LM), Langacker (2001) postula que temos um enquadramento atencional que nos impóe uma janela única de atenção para a visualização simultânea de um conteúdo conceitual. Portanto, traçando um paralelo com a aquisição da LE, podemos supor que a produção das oraçóes subordinadas concessivas, foco de tratamento desta investigação, demandaria um esforço cognitivo ainda maior para serem contidas dentro de um único enquadramento

\footnotetext{
${ }^{1}$ Nota retirada por conter informações sobre o autor.

${ }^{2}$ Termo cunhado por Schmidt, 1986, referindo-se à percepção consciente de aspectos da língua estrangeira pelos aprendizes.
} 
atencional; além de abordarem mais de um conteúdo conceptual, esses seriam contrastantes. Essa suposição corrobora a constatação, ainda na pré-coleta, da dificuldade dos participantes de produzir espontaneamente um texto escrito de discurso mais complexo.

Além disso, por um princípio universal de processamento linguístico, as posições iniciais e finais de um enunciado são naturalmente mais salientes quando comparadas à posição do meio, posição frequente de conectivos. Segundo VanPatten et al. (2004, p. 13), "as formas na posição do meio de um enunciado, portanto, talvez não recebam recursos atencionais suficientes para que as conexões forma-significado sejam iniciadas [pelo aprendiz],"3 razão pela qual se escolheu neste estudo salientar os conectivos concessivos.

Em sua Noticing Hypothesis, Schmidt (1990) postula que apenas o que é percebido de forma consciente pelos aprendizes no insumo é o que efetivamente torna-se insumo absorvido. O que, nas palavras de Robinson (2003, p. 654), mostra que Schmidt acredita serem a atenção focal e a percepção consciente - noticing - isomórficos. Schmidt $(1990,2001)$ sugere que não existe aprendizagem sem uma atenção consciente ${ }^{4}$ e focada ao insumo, sendo o noticing essencial para que haja o registro, o processamento e a posterior aquisição dos itens linguísticos. Esse autor, após detalhada análise das pesquisas disponíveis sobre o tema 'atenção e aprendizagem,' propõe uma versão mais forte de sua Noticing Hypothesis, postulando que, "ainda que haja percepção subliminal, não há uma aprendizagem subliminal." 5 Como o construto atenção não se refere a um único mecanismo, mas a um conjunto de sistemas interligados, que têm a função de controlar o processamento das informações recebidas pelo aprendiz, Schmidt conclui que a aprendizagem ocorre como um efeito colateral do insumo que foi identificado e processado intencionalmente (2001). Portanto, noticing é o primeiro passo na construção de uma língua, e não o final do processo. Sobre o papel do noticing, BleyVroman (1997) acredita que os aprendizes, ao trabalhar a sintaxe de uma segunda língua, ao contrário da primeira, não refazem seus parâmetros baseados

\footnotetext{
3 "Forms in middle positions, therefore, may not receive sufficient attentional resources to initiate FMCs (form-meaning connections)” (VANPATTEN, 2004, p. 13).

${ }^{4}$ Awareness.

5 "I have proposed a strong version of the 'noticing hypothesis, ' claim that while there is subliminal perception, there is no subliminal learning." (SCHMIDT, 2001, p. 26)
} 
em aspectos abstratos, mas o fazem a partir de padrões ou estruturas já acumulados, e o noticing torna-se a interface entre o insumo e o desenvolvimento de tais estruturas.

A atenção seletiva a aspectos salientados poderá se configurar em novos insights, em novas sistematizações, ou 'unidades linguísticas', suficientemente automatizadas para competir com os esquemas da LM em nível de igualdade (ACHARD, 1997; ACHARD; NIEMEIER, 2004). Essas unidades poderão originar novos mapeamentos, que eventualmente poderão se entrincheirar na memória de longo prazo, ficando disponíveis para futura utilização (TALMY, 2000; ELLIS, N., 1999).

Novos esquemas necessitam de muita prática comunicativa, através da execução de tarefas / atividades na instrução formal, para que sejam fortalecidos. Dessa forma, os aprendizes de uma LE poderão relacionar as novas formas linguísticas com seu significado, ou mesmo reativar chunks $^{6}$ de informação que já existam e estejam no nível semiativo de conscientização (CHAFE, 1994). Para Skehan (1998, p. 135), "é imperativo que as tarefas sejam sequenciais, já que os critérios que definem esta seqüência serão determinantes para a alocação de recursos atencionais necessários para o cumprimento destas tarefas." Além disso, as tarefas que exigem um alto nível de comunicação e processamento cognitivo podem também levar os aprendizes a melhorar a sua produção (SWAIN, 1995), estender a sua interlíngua ${ }^{7}$ (IL), e também usar o 'esforço mental' adicional em uma produção que poderá inclusive ocasionar a desestabilização de formas fossilizadas (ROBINSON, 2001).

As considerações acima nos remetem à postulação de Vygotsky (1962) de que a aprendizagem está ancorada no contexto social e cultural. São as interações dos indivíduos com esse contexto, por meio de suas experiências, que resultam no desenvolvimento cognitivo dos participantes. Portanto, a instrução formal apoiada em trabalhos de grupos, com participantes que se ajudariam na execução de tarefas ou resolução de problemas (ELLIS, 1994, 1997), poderia eficazmente atuar na reformulação da IL desses aprendizes. Swain (1998) afirma que os professores podem ser, assim, os responsáveis pela maximização de oportunidades para que determinadas estruturas, ao serem usadas de maneira comunicativa, captem a atenção dos aprendizes. Recomendação apoiada na afirmação de SKEHAN; FOSTER (2001, p. 185), que acreditam

\footnotetext{
${ }^{6}$ Grupamentos de palavras.

7 Tradução de Interlanguage - termo cunhado por Selinker (1972, p. 213).
} 
ter o professor o papel de "guiar os aprendizes para que percebam quais são as estruturas necessárias para a execução de determinada tarefa." 8

Nesse papel, o professor seria responsável pela reiterada exposição dos aprendizes ao insumo-alvo, o que poderia criar condições para uma consolidação fonológica e semântica dessas ocorrências na memória de longo prazo (ELLIS, N., 2001; 2006). Por meio de comparações entre o insumo e a sua produção linguística, esses aprendizes poderiam, de maneira consciente e objetiva, alterar gradualmente a sua IL (DOUGHTY, 2001), transformando sua produção em insumo para uma nova reformulação linguística (SCHMIDT, 1990; ELLIS, 2002).

\section{QUADRO 1}

Influências sobre o noticing

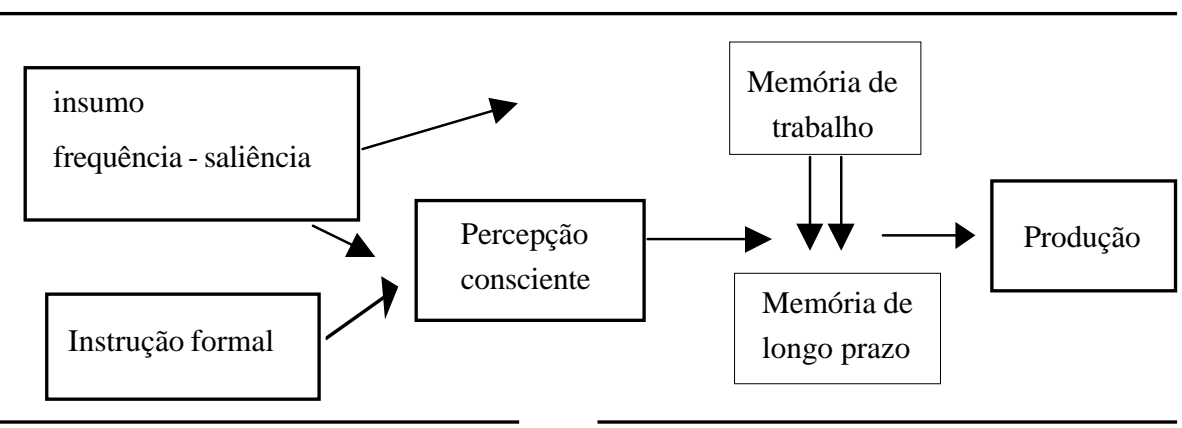

Fonte: SKEHAN, 1996, p.49.

No modelo acima (QUADRO 1), Skehan (1996) coloca o noticing como mediador entre o insumo recebido e a operacionalização dos sistemas de memória. Esse autor considera de central importância a saliência e a frequência da exposição ao insumo, fatores que influenciarão o sistema em desenvolvimento da IL do aprendiz, propiciando a criação de novos mapeamentos entre forma e sentido das estruturas na LE, e alteração da produção.

\section{Participantes}

A pesquisa foi realizada dentro do projeto EDUCONLE, ${ }^{9}$ parte do Programa Interfaces da Formação em Línguas Estrangeiras da FALE-UFMG,

8 "The role of the teacher is [...] to help them notice what language is required to do a particular task..."

${ }^{9}$ EDUCONLE: Educação Continuada de Professores de Língua Estrangeira. 
programa de educação continuada que teve início em 2002. O grupo de participantes foi constituído por 28 professores de inglês da rede pública municipal e estadual de Belo Horizonte e área metropolitana, exercendo a profissão em escolas de ensino médio e fundamental, com idades entre $30 \mathrm{e}$ 55 anos, graduados em Letras em faculdades do Estado de Minas Gerais.

O grupo foi distribuído em 6 subgrupos, cada um deles constituído por 4 alunos, alocados de forma aleatória (sorteio) aos grupos de controle (GC) e de tratamento (GT), necessários para a implementação de um experimento verdadeiro e uma análise quantitativa dos resultados. As intervençôes, as inúmeras atividades e as coletas de dados no decorrer das várias fases do tratamento só foram possíveis devido ao trabalho colaborativo entre duas professoras-em-treinamento - monitoras - que ficaram responsáveis pelo GC, enquanto esta pesquisadora assumiu o papel de professora do GT.

\section{Contexto pedagógico: instrumentos e dados}

A decisão de obter dados de um corpus escrito foi feita pela estreita relação da escrita com a maior possibilidade de percepção e conscientização dos aprendizes ao foco do tratamento. A escrita demanda mais planejamento e reflexão sobre a língua-alvo do que a fala, estando disponível para revisão e correção, fator fundamental para uma futura reestruturação e reformulação da IL. A essa se seguiu a definição do tema 'meio ambiente' subjacente à prática pedagógica, que contemplou os interesses e objetivos reais dos participantes da investigação. A abordagem adotada nas aulas de intervenção foi ancorada nos princípios comunicativos (HYMES, 1972;10 WIDDOWSON, 1978; HALLIDAY, 1973; CANALE; SWAIN, 1980) e na definição de tarefa ${ }^{11}$ como:

um trabalho de sala de aula que envolve os aprendizes na compreensão, manipulação, produção ou interação na língua-alvo enquanto sua atenção está preferencialmente focada no significado e não na forma (NUNAN, 1989).

\footnotetext{
${ }^{10}$ Hymes (1972) cunhou o termo competência comunicativa, objetivo maior da aprendizagem de LI, para ele resultante em parte do acúmulo de conhecimento social e cultural dos interlocutores, e responsável pela compreensão entre eles.

11 Também denominada 'atividade' por mim.
} 
A integração e o sequenciamento das atividades durante as etapas do tratamento foram então possíveis por terem sido ancoradas na produção da revista Environmental Issues.

\section{Foco do tratamento e corpus da coleta de dados}

Os resultados da pré-coleta de dados, obtidos do artigo inicial sobre a poluição ambiental urbana (FIG. 1) na LI, forneceram indicativos de aparente estagnação no processo desenvolvimental da IL dos participantes em algumas áreas consideradas de maior complexidade sintática e semântica. Ao utilizarem um registro mais formal na comunicação escrita, uma dessas áreas se sobressaiu pela sua quase completa ausência do discurso textual: o uso de períodos subordinados, mas, principalmente, a inexistência de conectivos. Apesar de os 24 alunos terem produzido um texto com opiniōes pessoais sobre reciclagem e preservação do meio urbano ${ }^{12}$ - um convite ao uso natural de conectivos de contraste - foram identificadas apenas duas ocorrências.

Incluindo esse artigo da pré-coleta, cada subgrupo (1-6) produziu um exemplar de uma revista sobre o meio ambiente. Esse trabalho foi subdividido em subtemas (QUADRO 2): contextualização do tema, constatação e análise dos problemas existentes, mais informação sobre o tema proposto, conscientização da extensão global do problema, e propostas para uma possível solução.

\section{QUADRO 2}

Descrição das etapas e temas das coletas de dados

\begin{tabular}{|c|c|}
\hline Pré-teste: & Contextualização: meio ambiente e reciclagem \\
\hline \multicolumn{2}{|c|}{$\begin{array}{l}\text { Aula sem coleta: } \text { Mais insumo: recursos naturais brasileiros: } \\
\text { o Pantanal e a Amazônia, a floresta tropical e o aquecimento global }\end{array}$} \\
\hline Coleta 2: & $\begin{array}{l}\text { Conscientização: a crise da água, poluição e espécies animais } \\
\text { ameaçadas no mundo }\end{array}$ \\
\hline Coleta 3: & $\begin{array}{l}\text { Solução: respeito ao planeta } \\
\text { Intervalo: Outros trabalhos + férias ( } 6 \text { semanas) }\end{array}$ \\
\hline Coleta 4: & $\begin{array}{l}\text { Novo tema: Dia dos Pais - Os papéis das mães e pais na } \\
\text { sociedade contemporânea }\end{array}$ \\
\hline
\end{tabular}

${ }^{12}$ Leitura para pré-coleta de dados: COLLINS, J. Radical plans for waste could start a big clean-up. The Guardian Weekly, 2002. Disponível em: <www.onestopenglish.com>. Acesso em: 22 abr. 2006. 
A elaboração de exemplares da revista Environmental Issues (QUADRO 3) foi responsável pela produção escrita de 2 artigos: pré-coleta e coleta 3, e 2 redações argumentativas (comparativa /contrastiva) - coletas 1 e 2. Já a coleta 4, coleta tardia, foi obtida de um artigo sobre o Dia dos Pais. As revistas, com formato preestabelecido e número igual de páginas, concorreram entre si à oportunidade de publicação on-line no site do projeto EDUCONLE. ${ }^{13}$ Essa premiação teve como objetivo principal motivar ainda mais os participantes a produzirem seus textos dentro dos critérios estabelecidos para as publicaçóes, e com a maior acuidade possível dentro da sua competência linguística.

QUADRO 3

Desenho da revista: Environmental Issues e alocação de tempo aos diversos tratamentos

\begin{tabular}{|c|c|c|c|c|c|}
\hline $\begin{array}{l}\text { Names of } \\
\text { group } \\
\text { participants }\end{array}$ & & Point of view & $\begin{array}{c}\text { Periscope: } \\
\text { points of } \\
\text { interest }\end{array}$ & $\begin{array}{l}\text { Points to } \\
\text { ponder }\end{array}$ & $\begin{array}{l}\text { Future } \\
\text { Scenario }\end{array}$ \\
\hline \multirow{6}{*}{$\begin{array}{l}\text { Name } \\
\text { of } \\
\text { magazine }\end{array}$} & $\begin{array}{l}\text { Free } \\
\text { Title }\end{array}$ & $\begin{array}{c}\text { Urban } \\
\text { Contrast }\end{array}$ & & $\begin{array}{l}\text { Cry for } \\
\text { help }\end{array}$ & $\begin{array}{l}\text { Looking } \\
\text { ahead }\end{array}$ \\
\hline & (29 abril) & $\begin{array}{c}\text { argumentative } \\
\text { essay } \\
\text { (13 de maio) }\end{array}$ & $\begin{array}{l}\text { drawings } \\
\text { pictures } \\
\text { diagrams } \\
\text { (20 maio) }\end{array}$ & $\begin{array}{c}\text { argumentative } \\
\text { essay } \\
\text { (27 de maio) }\end{array}$ & (3 de junho) \\
\hline & - & Tratamento 1 & - & Tratamento 2 & Tratamento 3 \\
\hline & - & $85 \mathrm{~min}$ & - & $40 m+30 m$ & $35 \mathrm{~min}$ \\
\hline & Pré-coleta & Coleta 1 & 一 & Coleta 2 & Coleta 3 \\
\hline & \multicolumn{4}{|c|}{ Fase 1} & Fase 2 \\
\hline
\end{tabular}

Desenho da revista: Environmental Issues e alocação de tempo aos diversos tratamentos

\section{Tratamento}

O desenho da pesquisa contemplou um sequenciamento de tarefas / atividades, dentro de uma abordagem FnaF proativa, que objetivaram guiar e direcionar o foco atencional dos aprendizes, alternadamente, do significado à forma, e destes para significados mais complexos. O tema 'meio ambiente' e o material autêntico para leitura, usado como fonte de informação, apoio à produção escrita e às atividades de sala de aula, foram os mesmos para as aulas

${ }^{13}$ http://www.letras.ufmg.br/educonle. Acesso: em 14 nov. 2006. 
do GC e GT. As três primeiras coletas foram obtidas em salas de aula separadas, enquanto a coleta tardia - coleta 4 - foi obtida ao término de uma aula regular, com a participação de todos os alunos, e sem trabalho linguístico com os conectivos-alvo. É importante ressaltar que, após a coleta 3, os grupos foram reunidos novamente para uma troca de informaçóes sobre o material das aulas do período em que estiveram em grupos separados. Um representante de cada grupo assumiu o papel de professor na explicação e correção das atividades. Essa foi a única oportunidade dos alunos do GC em sala de aula para trabalhar e perceber de forma consciente os conectivos concessivos - foco de tratamento do GT - e reativar seu conhecimento sobre o assunto.

Para que a pesquisa possa ser claramente compreendida, o QUADRO 4 apresenta uma compilação das fases da investigação e suas etapas, permitindo ao leitor ter uma visão global do processo:

\section{QUADRO 4}

Detalhamento do desenho da pesquisa

\begin{tabular}{|c|c|c|}
\hline $29 / 4$ & Pré-teste & $\begin{array}{l}\rightarrow \text { Grupo A e Grupo B } \rightarrow \text { produção escrita: artigo inicial da } \\
\text { Revista - título livre (texto } 1 \text { ) }\end{array}$ \\
\hline \multicolumn{3}{|r|}{ Intervalo: 2 semanas } \\
\hline \multirow[t]{2}{*}{$\begin{array}{l}13 / 5 \\
\text { Trat }^{14} 1\end{array}$} & \multirow[t]{2}{*}{ Coleta 1} & $\begin{array}{l}\rightarrow \text { Grupo A: atividades regulares com o mesmo conteúdo } \\
\text { temático do grupo B }\end{array}$ \\
\hline & & $\begin{array}{l}\rightarrow \text { Grupo B: atividades controladas de realce do insumo }{ }^{15}- \\
\text { percepção consciente do foco linguístico selecionado } \\
\text { Grupos A e B } \rightarrow \text { produção escrita: artigo } \\
\text { Urban contrast (texto } 2 \text { ) } \\
\text { (Correção colaborativa em pares - reescrita texto } 2 \text { ) }\end{array}$ \\
\hline $\begin{array}{l}20 / 5 \\
\text { Aula } 3\end{array}$ & & $\begin{array}{l}\rightarrow \text { Grupos A e B } \rightarrow \text { atividades regulares } \\
\rightarrow \text { Escolha do lay-out da revista: título e demais detalhes }\end{array}$ \\
\hline & Intervalo: 2 semanas \\
\hline $\begin{array}{l}27 / 5 \\
\text { Trat } 2\end{array}$ & Coleta 2 & $\begin{array}{l}\rightarrow \text { Grupos A: atividades regulares com o mesmo conteúdo } \\
\text { temático do grupo B } \\
\rightarrow \text { Grupo B: atividades controladas de realce do insumo - } \\
\text { percepção consciente do foco linguístico selecionado } \\
\text { Grupos A e B } \rightarrow \text { produção escrita: artigo } \\
\text { Cry for Help (texto 3) }\end{array}$ \\
\hline & & Intervalo: 1 semana \\
\hline
\end{tabular}

\footnotetext{
${ }^{14}$ Tratamento.

${ }^{15}$ Input enhancement.
} 


\begin{tabular}{l|l|l}
\hline $\begin{array}{c}\text { 3/6 } \\
\text { Trat 3 }\end{array}$ & Coleta 3 & $\begin{array}{l}\rightarrow \text { Grupo A: atividades regulares com o mesmo conteúdo } \\
\text { temático do grupo B } \\
\rightarrow \text { Grupo B: atividades controladas de realce do insumo - } \\
\text { percepção consciente do foco linguístico selecionado } \\
\text { Grupos A e B ? produção escrita: artigo } \\
\text { Let's save the Planet (texto 4) }\end{array}$ \\
\hline $12 / 8$ & Pós-teste & $\begin{array}{l}\text { Intervalo: 10 semanas } \\
\text { (Coleta 4 - tardia) } \\
\text { Grupos A e B ? produção escrita: artigo Father's and mother's roles in } \\
\text { contemporary society } \\
\text { (texto 5) }\end{array}$ \\
\hline Legenda: Grupo A - Grupo de Controle \\
Grupo B - Grupo de Tratamento \\
Trat - Tratamento
\end{tabular}

\section{Tratamento 1}

Esta primeira intervenção no GT teve a duração de 85', dividida entre três atividades distintas. Ao final, foi produzido o primeiro texto pelos dois grupos: o artigo intitulado Urban Contrast (QUADRO 3 -Coleta 1).

\section{Etapa 1:}

- Atividade 1a: foi feita após um breve brainstorming dos textos prescritos como leitura extensiva. Após um levantamento do conteúdo temático, os participantes identificaram, realçaram os conectivos concessivos de um dos textos $^{16}$ e refletiram sobre a sua função.

- Atividade 1b: após o reconhecimento pontual, os participantes trabalharam com oraçōes retiradas do texto inicial e do texto "A Stinking Mess", ${ }^{17}$ transcritas em uma lâmina (retroprojetor) com espaços para serem completados oralmente pelo grupo.

Etapa 2: prática oral e preparação I para a produção escrita. Os alunos receberam vários pares de fotos autênticas ${ }^{18}$ para que comparassem e

${ }^{16}$ SHEA, M. The Big Recycling Scandal. Speak Up, Rio de Janeiro, ano 9, n. 117, p. 4-6, dez. 1996.

${ }^{17}$ LANGONE, J. A Stinking Mess. Time International - Planet of the Year, New York, n. 1, p. 26-28, jan. 1989.

18 WOOLCOTT, L. Take Your Pick: Activities for using photographs in language learning. Essex: Longman, 1992. 
contrastassem as imagens em pares, trocando-as dentro dos seus grupos. Essas imagens, duas delas de Belo Horizonte, mostravam cenários urbanos contrastantes: conservação e limpeza e abandono e lixo jogado em ruas, lagos e rios.

Etapa 3: 'leilão' e preparação II para a produção escrita: nesta etapa os participantes, em seus grupos, construíram orações subordinadas comparativas e / ou contrastantes, expressando suas ideias sobre a diversidade de cenários trabalhada oralmente na fase anterior.

\section{Tratamento 2}

A segunda intervenção, duas semanas após a primeira, teve a duração de 40 minutos (QUADRO 3). Houve a distribuição prévia, para leitura extensiva de uma série de $\operatorname{artigos}^{19}$ curtos sobre espécies de animais em perigo de extinção, crise da água e o combate à poluição. Os participantes tiveram também a oportunidade para a prática de andaimento durante 30 minutos. Essa interação entre pares dentro dos subgrupos ocorreu após a tarefa escrita: a redação Cry for help: inevitable destruction or sustainable growth?

Etapa 1: brainstorming do tema: instigados pela imagem, colocada na lâmina What On Earth Are We Doing.20 sobre as queimadas no Brasil, os participantes do GT emitiram suas opinióes sobre o fato e discutiram o assunto em seus grupos.

Etapa 2: reativação do foco: após a atividade anterior, cada grupo ficou responsável por reativar aspectos importantes de um dos temas lidos, fazendo anotaçóes dos pontos importantes para futura discussão.

- Atividade 2a: os participantes receberam gráficos ${ }^{21}$ relativos aos temas da leitura prévia, discutiram as informaçoos e fizeram suas anotações em uma tabela com 3 colunas: similar, different e synthesis. Eles analisaram os dados e combinaram as informações em períodos compostos, comparando e

${ }^{19}$ KIRBY, A. Changing earth: Part 1: Species under threat; Part 2: World water crisis; Part 5: Tackling pollution. Disponível em: <http://news.bbc.co.uk/2/hi/science/ nature>. Acesso em: 25 maio 2006.

${ }^{20}$ SANCTON, T. Torching the Brazilian Rainforest. Time International - Planet of the Year, New York, n. 1, p. 8-9, jan 1989.

${ }^{21}$ KIRBY, A. Changing earth: Part 1: Species under threat; Part 2: World water crisis; Part 5: Tackling pollution. Disponível em: <http://news.bbc.co.uk/2/hi/science/ nature>. Acesso em: 25 maio 2006. 
contrastando os quadros de poluição, falta de água, e números de animais em extinção ao redor do mundo. Em seguida, escolheram as melhores orações de cada grupo, que foram colocadas em lâminas e apresentadas à classe. Nesta fase, todos contribuíram na análise da adequação do uso das orações subordinadas.

- Atividade 2b: uma segunda atividade de conscientização e reforço do uso adequado dos conectivos foi feita utilizando-se duas baterias de exercícios. Houve grande colaboração entre os membros dos grupos.

Etapa 3: edição e reformulação do texto após a produção escrita: os participantes trocaram as redaçôes entre si em seus grupos de trabalho, e muitos colaboraram com seus pares na reformulação de partes do texto que consideraram inadequadas.

\section{Tratamento 3}

Esta terceira e última intervenção, uma semana após a segunda, teve a duração de 35 minutos. Como nas aulas de intervenção anteriores, os alunos receberam previamente o material de leitura: Respect the land, ${ }^{22}$ escrito por $\mathrm{Al}$ Gore. Foi dada uma nova oportunidade para a prática de andaimento e, logo após, foi feita uma apresentação oral no formato de um discurso breve. Esta sequência de atividades de curta duração em grupos culminou com a produção escrita final desta Fase 1 da coleta de dados - o artigo intitulado: Looking ahead: let's save the planet! (QUADRO 3).

\section{Coleta tardia}

Uma última coleta, a coleta 4 - Fase 2 - (QUADRO 3) foi feita para que fosse possível tentar estabelecer um panorama mais amplo sobre os efeitos dos tratamentos. Após um período de 10 semanas, incluindo um período de 3 semanas de férias escolares, os alunos receberam novo material ${ }^{23}$ autêntico de leitura como apoio para mais uma produção escrita: Help wanted, a father for every family, Mothering e Who are fathers? Os alunos foram divididos em três

\footnotetext{
${ }^{22}$ GORE, Al. Respect the Land. Time Special Issue, New York, p. 8-9, nov. 1997. ${ }^{23}$ http://fredericksburg.com/News/FLS/2006/062006/06042006/194861; http:// www.fathersdirect.com/index.php?nID=43; http://parenthood.library.wisc.edu/ Weissbourd/Weissbourd.html.
} 
grupos e cada um recebeu um texto diferente para que houvesse uma motivação comunicativa para o trabalho de brainstorming antes da produção escrita individual. Desta feita, o tema escolhido para a redação foi o 'Dia dos Pais'.

\section{Discussão dos resultados}

As ocorrências dos conectivos foram classificadas de acordo com o grau de adequação às construções equivalentes na língua-alvo. Foram atribuídos valores para que fosse possível a obtenção de um resultado quantitativo (TAB. 1).

TABELA 1

Categorização dos dados da investigação

\begin{tabular}{|c|c|c|c|c|c|}
\hline Ocorrências & & \multicolumn{2}{|c|}{ Categoria } & Valor & Inadequações \\
\hline $\begin{array}{l}\text { Convergentes } \\
\text { com a } \\
\text { Língua-alvo }\end{array}$ & $\begin{array}{l}\text { forma + função } \\
\text { semelhantes à } \\
\text { língua-alvo }\end{array}$ & \multicolumn{2}{|c|}{ CLA } & 1.00 & -.-.--.- \\
\hline \multirow{3}{*}{$\begin{array}{l}\text { Divergentes } \\
\text { da } \\
\text { Língua-alvo }\end{array}$} & forma inadequada & \multirow[b]{3}{*}{ DLA } & FOI & 0.75 & $\begin{array}{l}\text { despite + of } \\
\text { in spite of / although } \\
+ \text { to-infinitive }\end{array}$ \\
\hline & \multirow[t]{2}{*}{$\begin{array}{l}\text { função inadequada } \\
\text { (in spite of } \mathrm{x} \text { although) }\end{array}$} & & FUI & 0.50 & $\begin{array}{l}\text { in spite of / despite + SVO } \\
\text { although + substantivo / that }\end{array}$ \\
\hline & & & CFD & 0.25 & while, whereas \\
\hline
\end{tabular}

Apesar das ocorrências divergentes (FOI, FUI, e CFD) apresentarem um grau de distanciamento diferente da língua-alvo, essas foram agrupadas sob a sigla DLA, formando um grupo para comparação com as ocorrências convergentes com a língua-alvo (CLA). Abaixo estão alguns exemplos ilustrativos:

Categoria CLA:

1) Although I disagree with this policy...

2) ... even if we can't pay higher taxes...

3) ... in spite of this, the ecosystems are...

4) ... beautiful landscapes to see while we are passing...

5) ... despite the horrible actions of the government people

6) ... whereas governments have to be concerned... 


\section{Categoria FOI:}

1) ... despite of brilliant and helpful solutions, Earth's problems are...

2) Despite of paying taxes for the government...

3) ... although our city to be called 'Belo Horizonte'...

Categoria FUI:

1) Despite they know this is a bad habit...

2) In spite of authorities spend a lot of money in aid...

3) ... although all these problems, people are...

\section{Categoria CFD:}

1) Whereas their practice of these activities we are creating...

2) ... his own experience while a child in his family's farm.

Os gráficos (GRAF. 1 e 2) mostram, em números absolutos, a importância e o impacto do tratamento nos resultados do GT. O número de ocorrências nas redações desse grupo reflete de maneira expressiva a importância de este trabalho ter ocorrido dentro de uma abordagem pedagógica que permitiu e ofereceu oportunidades para que esses participantes pudessem perceber conscientemente os conectivos concessivos, seu papel significativo no discurso escrito nos gêneros escolhidos - artigo e redação argumentativa - e pudessem, por tentativas e acertos, passar a usá-los e, possivelmente, reestruturar sua IL.

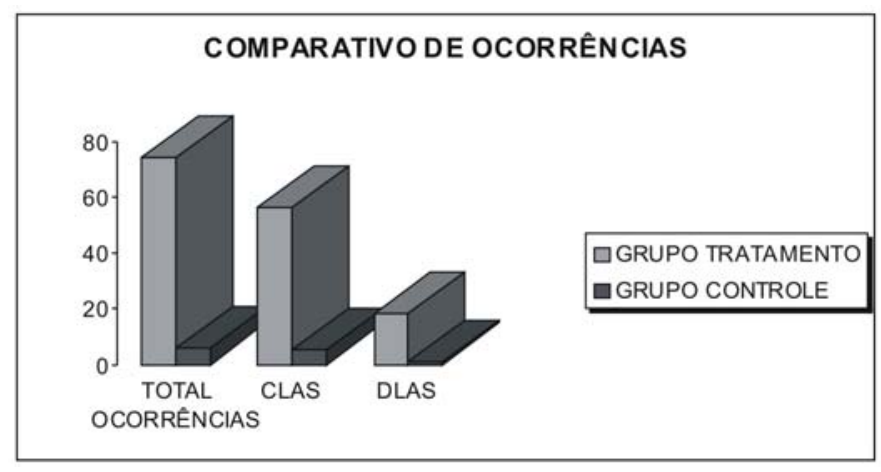

GRÁFICO 1 - Quadro comparativo entre os GT e GC

Os resultados da coleta 1 (GRAF. 2) mostram que $1 / 3$ das ocorrências foi de enunciados divergentes da língua-alvo (DLA), número equivalente a $33,4 \%$ do total, enquanto o número de enunciados convergentes (CLA) foi de $66,7 \%$. Na coleta 2 (GRAF. 2), enquanto as DLAs recuaram para um 
patamar de 18,2\% em relação ao número total de ocorrências, a porcentagem de CLAs aumentou para $81,8 \%$, uma forte indicação do efeito positivo cumulativo das atividades geradoras de percepção consciente. A coleta 3 (GRAF. 2) indica a porcentagem de $75 \%$ para as CLAs e de $25 \%$ para as DLAs. Na coleta tardia (GRAF. 2), não é encontrada nenhuma ocorrência de uso divergente da língua-alvo, um fato bastante promissor.

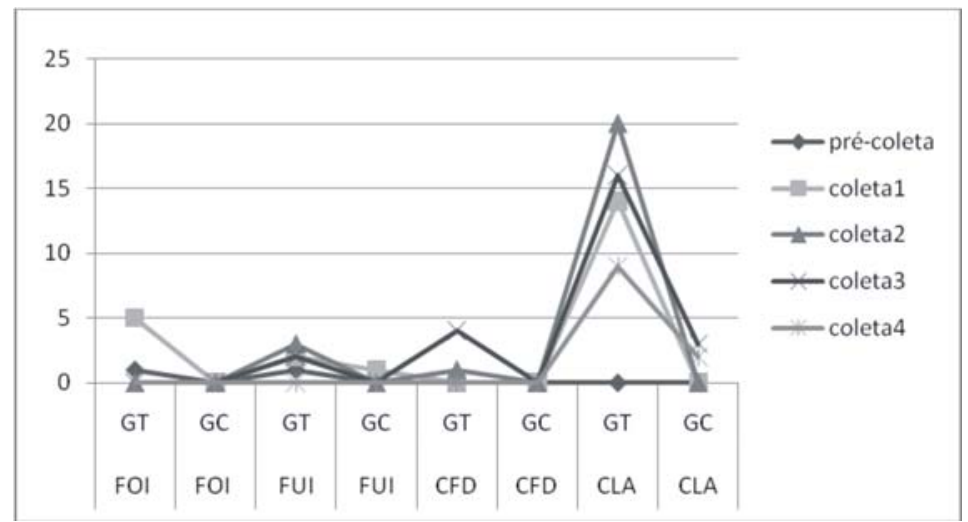

\section{GRÁFICO 1 - Ocorrências convergentes (CLAs) e divergentes FOI - FUI - CFD (DLAs)}

Minha análise é a de que, na coleta 3, após a motivação inicial dos participantes de incluírem as novas combinações linguísticas nos textos, fato identificado nas coletas 1 e 2 , há um início de estabilização das reformulações que poderiam estar ocorrendo na IL. Nessa coleta, há evidência de diferença significativa (TAB. 2) entre o GT e GC ao nível de significância de 0,05 (p_valor=0,02):

TABELA 2

Resultado do teste não-paramétrico Wilcoxon Signed Ranks

\begin{tabular}{l|l|l|l|l}
\multicolumn{2}{l}{ Região de rejeição } \\
\hline \multirow{2}{*}{ Ocorrências } & & Comparação & $|Z|=1,96$ & $\mathrm{p}<0,05$ \\
\hline \multirow{2}{*}{ CLA } & & & p_valor \\
\cline { 4 - 5 } & \multirow{2}{*}{ Coleta 3 } & Tratamento - Controle & $-2,36$ & 0,02 \\
\cline { 3 - 5 } & & & & \\
\hline
\end{tabular}


O patamar de ocorrências ainda é alto, mas começa a apresentar uma pequena tendência de queda, sugerindo um movimento em direção ao uso dos conectivos de maneira menos exacerbada, mais convergente com os números que seriam encontrados em textos semelhantes na língua-alvo.

Ao compararmos a evolução das ocorrências em números absolutos nos dois grupos, encontramos uma diferença significativa, o que indica claramente a importância de uma prática pedagógica com atividades geradoras de percepção consciente. Após as três aulas de intervenções, foram analisadas as redaçôes de todas as coletas dos participantes, em um total de 120 textos, incluindo a pré-coleta, considerada como o pré-teste deste experimento, e a coleta tardia 4 - o pós-teste. Ao todo, foram encontrados 80 enunciados-foco, que se distribuíram entre os dois grupos da seguinte forma (TAB. 3):

TABELA 3

Distribuição das ocorrências dos enunciados-foco do tratamento $(G T+G C)$

\begin{tabular}{l|c|c|c|c|c|c}
\hline & Pré-coleta & Coleta 1 & Coleta 2 & Coleta 3 & Coleta 4 & Total \\
\hline Redações & 24 & 24 & 24 & 24 & 24 & 120 \\
\hline CLÃS & 0 & 14 & 18 & 18 & 11 & 61 \\
\hline DLAs & 2 & 8 & 4 & 5 & 0 & 19 \\
\hline
\end{tabular}

Uma análise da tabela acima nos possibilita a constatação de que os enunciados convergentes com a língua-alvo (CLAs) foram produzidos em número muito superior aos enunciados divergentes da língua-alvo (DLAs); ou seja, de um total de 80 enunciados, 61 deles ou 76, 25\% foram CLAs, enquanto apenas $23,75 \%$ foram DLAs.

O gráfico abaixo (GRAF. 3) mostra a evolução das CLAs e a redução do uso das DLAs nos GT e GC, coleta a coleta, ilustrando de maneira significativa o efeito das atividades de percepção consciente no aumento da produção de enunciados com os conectivos. Ele também mostra os resultados absolutos dos dois grupos, nos quais é possível constatar a evolução positiva mais significativa do GT: 


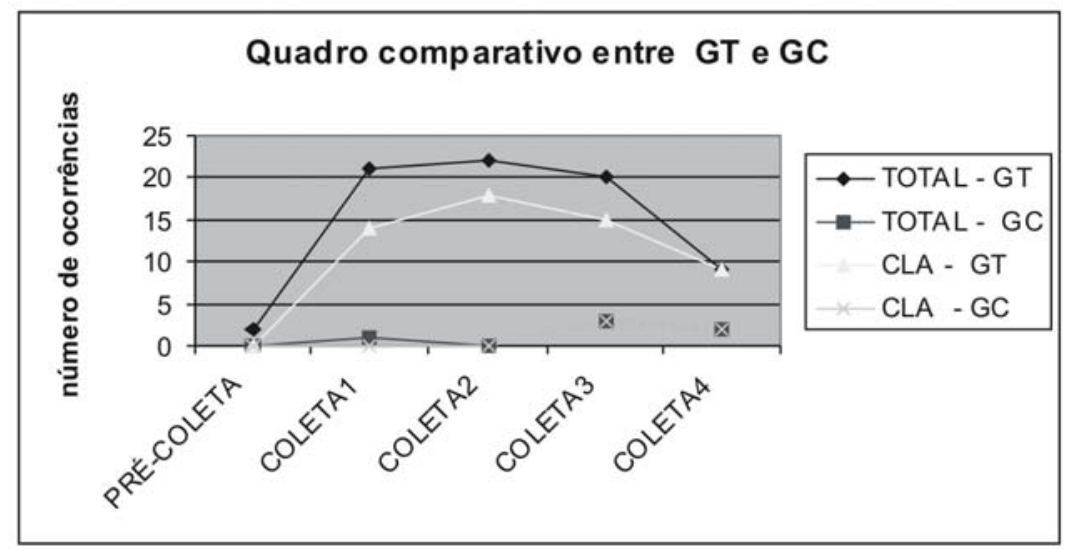

GRÁFICO 3 - Número de ocorrências convergentes e relação com o número total em cada coleta

E, para finalizar, o GRAF. 4 mostra, em percentuais, a evolução moderada e ascendente da reformulação linguística da IL dos participantes do GT, coleta a coleta:

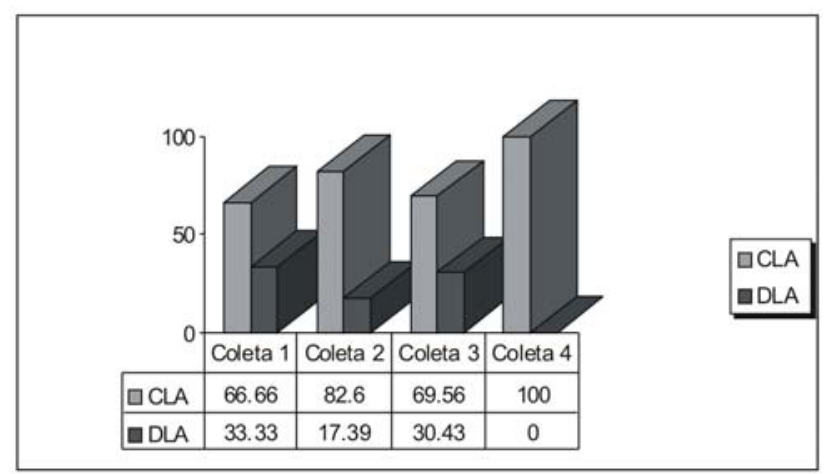

GRÁFICO 4 - Comparativo (\%) entre as categorias CLA e DLA na seqüência de coletas nos GT

\section{Conclusão}

Várias leituras desse processo investigativo tornam-se possíveis ao observarmos os dados mostrados nos vários gráficos e tabelas incluídos na seção anterior. Dentre elas, a da análise dos efeitos do direcionamento do foco atencional dos aprendizes na aprendizagem da LE e no aumento da produção 
linguística, e a da análise das condições da prática pedagógica, incluindo as atividades e a sua interligação, devido ao tema das tarefas. A primeira e mais relevante de todas seria a confirmação de algumas postulaçõos de linguistas cognitivos, citados neste trabalho, pelos resultados estatísticos e numéricos, obtidos após os tratamentos que visaram (re)direcionar o foco atencional dos participantes aos conectivos concessivos - itens gramaticais com maior opacidade em um texto escrito mais complexo.

Ancorados na literatura disponível pesquisada, podemos supor que o aprendiz produza períodos simples, equivalentes a uma unidade atencional (CHAFE, 1994) ou ao conteúdo conceptual de um único enquadramento atencional (LANGACKER, 2001) com maior facilidade cognitiva. E, ancorados nos resultados, também parece ser mais fácil a produção de períodos por coordenação que contenham significados semelhantes a períodos compostos por subordinação (TALMY, 2000). Uma possível justificativa à predominância de períodos simples ou coordenados, identificados nos textos, também poderia ser apoiada em Langacker (2001), quando sugere que a compressão de mais unidades conceptuais (entenda-se períodos subordinados) em uma única 'janela de atenção' reduz a saliência que esses períodos teriam como oraçōes simples.

A relevância do papel da 'atenção', quando direcionada a um campo atencional específico no decorrer das atividades, também parece indiscutível. A percepção consciente do papel dos conectivos while, whereas, although, even if, despite e in spite of pelos participantes ficou demonstrada na reformulação linguística que foi sendo efetuada a cada nova produção escrita. Após os Tratamentos 1 e 2, a percepção consciente das relações subordinadas é evidenciada na produção escrita, em que foi possível observar um aumento expressivo não só da utilização desses conectivos concessivos como também da complexificação do discurso. Enquanto no pré-teste não foram identificadas ocorrências convergentes com a língua-alvo (CLAs), e apenas duas ocorrências divergentes da língua-alvo (DLAs), o equivalente a 100\% de erros, no pós-teste foram identificadas apenas ocorrências convergentes (CLAs), ou 100\% de acertos, sugerindo uma reformulação da IL já no curto prazo. Portanto, o direcionamento da 'atenção' de aprendizes ao que lhes é menos saliente parece ser uma das decisóes necessárias que devem ser repensadas pelos professores em sua prática pedagógica (GRUNDY, 2004, p. 137).

Essa decisão foi tomada durante o processo investigativo, quando do planejamento de um conjunto coordenado de atividades / tarefas interligadas 
tematicamente, que foram executadas para obtenção dos dados. Os resultados sugerem que os participantes puderam se beneficiar de forma significativa com as intervençōes pontuais, proativamente planejadas para contemplar as janelas de oportunidades cognitivas que foram se abrindo durante o processo. A análise dos dados mostra a importância de se incluir atividades / tarefas captadoras do foco atencional dos aprendizes adultos a áreas que se apresentam opacas, sejam por desconhecimento ou pela pouca visibilidade no cotidiano dos aprendizes. Implementadas com frequência, as oportunidades contextualizadas os conduzem de forma natural a utilizar a LE de maneira mais apropriada e espontânea. Segundo Schmidt (1986), não há aprendizagem sem essa percepção (VANPATTEN, 1998). E se a nossa atenção é seletiva (BROADBENT, 1958), pode-se inferir que só é apreendido aquilo que é selecionado pelos indivíduos (ELLIS, N., 1999).

Além disso, a oferta de insumo interligado tematicamente e de forma sequencial durante o tratamento pode ter desempenhado um papel importante para a exposição recorrente dos participantes ao foco de tratamento. Porém, esse efeito positivo só foi identificado no GT em que houve um trabalho pontual com os conectivos, e não no GC, apesar de o material de leitura exposição incidental ao foco - ter sido o mesmo. Como alguns autores cognitivistas sugerem, numa extensão do conceito de organização visual figura e fundo ${ }^{24}$ à linguagem, o que é lembrado é a figura, saliente, dinâmica, independente, a oração principal, e não o fundo, considerado a referência, o contexto estático, o pano de fundo, a oração subordinada. No caso do foco de tratamento, apenas a exposição incidental não foi suficiente para que os aprendizes do GC percebessem as relações de concessão, contraste e comparação, possivelmente situadas no fundo da sua janela atencional, e não no primeiro plano, como figura.

Uma outra faceta deste estudo também permitiu a observação do trabalho coletivo dos participantes em seus grupos, em que a oportunidade da prática do andaimento, objetivo secundário desta investigação, visou auxiliálos na reformulação de seus textos em duas oportunidades distintas: nas aulas do Tratamento 2 e do Tratamento 3. Apesar de ficar evidenciada a sua importância para o compartilhamento de opiniōes durante as interaçôes ${ }^{25}$ dos seus integrantes, a produção do GC dos enunciados-foco na coleta 2 foi nula,

\footnotetext{
${ }^{24}$ KOFFKA, 1935.

${ }^{25}$ Um dos pilares da Abordagem Comunicativa contemporânea.
} 
enquanto no GT foram identificadas 18 ocorrências; e na coleta 3, a produção do GC foi de 3 ocorrências, comparadas com 15 do GT, ou somente $20 \%$ do resultado do GT. Os resultados sugerem que a prática do andaimento, por meio da colaboração entre pares, por si só não aumenta as possibilidades de haver uma saliência de aspectos do insumo.

Como conclusão, e considerando o tamanho do corpus analisado neste estudo, outras investigações se fazem necessárias nessa direção promissora. Elas precisam ser feitas em novos contextos, com um número maior de participantes, e também com outros perfis, nos quais as conexôes forma-significado na LE poderão ser mais investigadas sob a luz das descobertas científicas mais recentes nas áreas de biologia, neurologia e psicologia. Uma possível continuação nessa linha de investigação poderia incluir a análise da otimização da prática pedagógica em um futuro muito próximo. $\mathrm{O}$ processo de aprendizagem da $\mathrm{LE}$ estará ainda mais aliado à tecnologia, ao letramento visual, e o foco de atenção dos aprendizes, hoje difuso e multidirecionado, precisará ser mais convergente e direcionado aos objetivos linguísticos na prática pedagógica. Naquele momento, o ensino da LE estará finalmente ligado de fato ao seu uso no mundo real, em tempo real, e em perfeita sincronia com o mundo multimídia do século 21, em que a imagem é o centro da nossa 'janela de atenção'.

\section{Referências}

ACHARD, M. Cognitive Grammar and SLA Investigation. Journal of Intensive English Studies, v. 2, p. 157-176, 1997.

ACHARD, M.; NIEMEIER, S. Cognitive Linguistics, Language Acquisition, and Pedagogy. In: ACHARD, M.; NIEMEIER, S. (Ed.). Cognitive Linguistics, Second Language Acquisition, and Foreign Language Teaching. Berlin: Mouton de Gruyter, 2004. p. 1-11.

BLEY-VROMAN, R. Features and Patterns in Foreign Language Learning. Research Forum, 2. Michigan, 1997, Proceedings.... Michigan, 1997.

BROADBENT, D. E. Perception and Communication. London: Pergamon Press, 1958.

CANALE, M.; SWAIN, M. Theoretical bases of communicative approaches to second-language teaching and testing. Applied Linguistics, v.1, n.1, p. 1-47, 1980 .

CHAFE, W. Discourse, Consciousness and Time. Chicago: University of Chicago Press, 1994. 
DOUGHTY, C. Cognitive underpinnings of focus on form. In: ROBINSON, P. (Ed.). Cognition and Second Language Instruction. Cambridge: CUP, 2001. p. 206-257.

ELLIS, N. Vocabulary Acquisition: The implicit ins and outs of explicit cognitive mediation. In: ELLIS, N. C. (Ed.). Implicit and explicit learning of languages. London: Academic Press, 1994.

ELLIS, N. Cognitive Approaches to SLA. Annual Review of Applied Linguistics, n. 19, p. 22-42, 1999.

ELLIS, N. Memory for Language. In: ROBINSON, P. (Ed.). Cognition and Second Language Instruction. Cambridge: CUP, 2001. p. 33-68.

ELLIS, N. Cognitive Perspectives on SLA: The Associative-Cognitive CREED. In: Bardovi-Harlig, K.; Dörnyei, Z. (Ed.). Themes in SLA Research. AILA Review 19, p. 100-121, 2006.

ELLIS, R. Second Language Acquisition. Oxford: OUP, 1997.

ELLIS, R. Interlanguage variability in narrative discourse: Style shifting in the use of the past tense. Studies in Second Language Acquisition, v. 9, n. 1, p. 1-20, 1987.

ELLIS, R. Does form-focused instruction affect the acquisition of implicit knowledge? Studies in Second Language Acquisition, v. 24, n. 2, p. 223-236, 2002. ELLIS, R. The Place of Grammar in the Second / Foreign Language Curriculum. In: HINKEL, E.; FOTOS, S. (Ed.). New Perspectives on Grammar Teaching in Second Language Classrooms. New Jersey: Lawrence Erlbaum Associates, 2002. p. 17-34.

GORE, Al. Respect the Land. Time Special Issue, New York, p. 8-9, nov. 1997

GRUNDY, P. The Figure / Ground Gestalt and Language Teaching Methodology. In: ACHARD, M.; NIEMEIER, S. (Ed.). Cognitive Linguistics, Second Language Acquisition, and Foreign Language Teaching. Berlin: Mouton de Gruyter, 2004. p.119-141.

HALLIDAY, M. A. K. Explorations in the Functions of Language. London: Edward Arnold, 1973.

HYMES, D. On communicative competence. In: PRIDE, J. B.; HOLMES, J. (Ed.). Sociolinguistics. Harmondsworth: Penguin, 1972.

KIRBY, A. Changing earth: Part 1: Species under threat; Part 2: World water crisis; Part 5: Tackling pollution. Disponível em: <http://news.bbc.co.uk/2/hi/science/ nature>. Acesso em: 25 maio 2006.

KOFFKA, K. Principles of Gestalt Psychology. New York: Hartcourt, Brace and Company, 1935. 
LANGACKER, R. Discourse in Cognitive Grammar. Cognitive Linguistics, n. 12, p. 143-188, 2001.

LANGONE, J. A Stinking Mess. Time International - Planet of the Year, New York, n. 1, p. 26-28, jan. 1989.

LONG, M. Focus on Form: A Design Feature in Language Teaching. In: BOT, K.; Ginsberg, R.; Kramsch, C. (Eds.). Foreign Language Research in CrossCultural Perspectives. Amsterdam: John Benjamins, 1991. p. 39-52.

NUNAN, D. Designing tasks for the Communicative Classroom. Cambridge: CUP, 1989.

PIENEMANN, M. Language Processing and Second Language DevelopmentProcessability Theory. Amsterdam: John Benjamins, 1998.

ROBINSON, P. Attention, Memory, and the 'Noticing Hypothesis' - Review article. Language Learning, n. 45, v. 2, p. 283-331, 1995.

ROBINSON, P. Task complexity, task difficulty, and task production: exploring interactions in a componential framework. Applied Linguistics, v. 22/1, p. 27-57, 2001.

ROBINSON, P. Attention and Memory during SLA. In: DOUGHTY, C.; LONG, M. (Ed.) The Handbook of Second Language Acquisition. Oxford: Blackwell Publishing, 2003. p. 631-678.

SANCTON, T. Torching the Brazilian Rainforest. Time International-Planet of the Year, New York, n. 1, p. 8-9, jan 1989.

SCHMIDT, R.; FROTA, S. Developing basic conversational ability in a second language: A case study of an adult learner of Portuguese. In: DAY, R. (Ed.). Talking to Learn: conversation in second language learning. Rowley, Mass: Newbury House, 1986. p. 237-326.

SCHMIDT, R. The role of consciousness in second language learning. Applied Linguistics, v. 11, n. 2, p. 129-158, 1990.

SCHMIDT, R. Attention. In: ROBINSON, P. (Ed.). Cognition and Second Language Instruction. Cambridge: CUP, 2001. p. 3-32.

SHEA, M. The Big Recycling Scandal. Speak Up, Rio de Janeiro, ano 9, n. 117, p. 4-6, dez. 1996.

SKEHAN A Framework for the implementation of Task-based instruction. Applied Linguistics, v. 17, n. 1, 1996.

SKEHAN, P. A Cognitive Approach to Language Learning. Oxford: OUP, 1998. SKEHAN, P.; FOSTER, P. Cognition and Tasks. In: ROBINSON, P. (Ed.). Cognition and Second Language Instruction. Cambridge: CUP, 2001. p. 183-205. 
SWAIN, M. Focus on Form through conscious reflection. In: DOUGHTY, C.; WILLIAMS, J. (Ed.). Focus on Form in Classroom Second Language Acquisition. Cambridge: CUP, 1998. p. 64-81.

SWAIN, M. Three functions of output in second language learning. In: Principle and Practice in Applied Linguistics: studies in Honour of H. G. Widdowson. Oxford: OUP, 1995. p. 125-144.

SWAIN, M.; LAPKIN, S. Problems in Output and the Cognitive Processes they generate: A Step Towards Second Language Learning. Applied Linguistics, v. 16, n. 3, p. 371-391, 1995.

TALMY, L. Toward a Cognitive Semantics. Cambridge: Massachusetts Institute of Technology, 2000.

TOMLIN, R.; VILLA, V. Attention in Cognitive Science and Second Language Acquisition. Studies in Second Language Acquisition, v. 16, p. 183-203, 1994.

VANPATTEN, B. Input Processing and Grammar Instruction in Second Language Acquisition. New Jersey: Ablex, 1996.

VANPATTEN, B.; WILLIAMS, J.; ROTT, S. Form-meaning Connections in Second Language Acquisition. In: VANPATTEN, B.; WILLIAMS, J.; ROTT, S.; OVERSTREET, M. (Ed.). Form-meaning Connections in Second Language Acquisition. Mahwah: Lawrence Erlbaum Associates, 2004. p. 1-26.

VANPATTEN, B. Language processing and Second language DevelopmentProcessability Theory. Amsterdam: John Benjamins Publishing Company, 1998. VYGOTZKY, L. Thinking and Speaking. Massachusetts: MIT Press, 1962. WIDDOWSON, H.G. Teaching Language as Communication. Oxford: OUP, 1978.

WOOLCOTT, L. Take Your Pick: activities for using photographs in language learning. Essex: Longman, 1992.

Recebido em 17 de maio de 2009. Aprovado em 19 de outubro de 2009 